\title{
ON THE DESIGN OF PRACTICAL ASYNCHRONOUS PHYSICAL LAYER NETWORK CODING
}

\author{
Francesco Rossetto* \\ DLR (German Aerospace Center) \\ Institute of Communications and Navigation \\ 82234 Weßling, Munich, Germany
}

\begin{abstract}
Decode-and-forward physical layer network coding is one of the most high-performing ideas for wireless network coding. However, all the present schemes work under rather ideal assumptions, such as synchronous reception of the colliding signals. This paper proposes a simple and practical system which removes many of the assumptions made in the past and also designs a soft-output demodulator for this type of network coding.
\end{abstract}

\section{INTRODUCTION}

The concept of physical layer network coding (PNC) has lately attracted a certain interest in the network coding and signal processing communities [1-9]. The basic idea can be illustrated in the two-way relay channel (Fig. 1). Two nodes (A and B) must exchange two packets (A's X and B's Y) through an intermediate relay R. In classic Network Coding (NC), A would send $X$ in time slot 0 , B would transmit $\mathrm{Y}$ in slot 1 and $\mathrm{R}$ would broadcast $\mathrm{X} \oplus \mathrm{Y}$ in slot 2 . In physical layer network coding, A and B would simultaneously send $\mathrm{X}$ and $\mathrm{Y}$, while $\mathrm{R}$ would relay a function of $\mathrm{X}$ and $\mathrm{Y}$, which is invertible in $\mathrm{X}$ or $\mathrm{Y}$ as soon as the other variable is known. Given that A and B know their own packets, they can each potentially decode the other node's frame. This approach would require 2 rather than 3 slots, with a $50 \%$ improvement in spectral efficiency over classic NC. Note that R need not decode X and Y separately, but it is enough to directly decode a linear combination $\mathrm{Z}$. This can potentially reduce the error rate because less information must be extracted from the received signal $[1,2,6]$. We highlight that PNC can be applied in a much wider range of scenarios than just the two way relay channel (TWRC) $[4,5]$. On the other hand, this paper will be focused on this setting because it is simple, includes all necessary elements of PNC, and enables to focus on signal processing issues.

Two main approaches have implemented this idea. In amplifyand-forward physical layer network coding (AF-PNC, also called analog network coding [1-5]), the relay amplifies the analog superposition of $\mathrm{X}$ and $\mathrm{Y}$ and broadcasts this signal, that will be called $S$. In addition, it embeds in the packet header the channel coefficients $h_{A}$ and $h_{B}$, which describe the channels from A to $\mathrm{R}$ and $\mathrm{B}$ to $\mathrm{R}$, respectively. Channel reciprocity is assumed; hence the channels from, for instance, $\mathrm{A}$ to $\mathrm{R}$ and from $\mathrm{R}$ to $\mathrm{A}$ are identical. The upshot is that both A and B have sufficient information to subtract their own signal from $S$. The resulting signal depends only on the intended packet, under the hypothesis of perfect cancellation. On the

${ }^{*}$ The first author performed the work while at the University of Padova, Italy. The second author is also with the California Institute for Telecommunications and Information Technology - UC San Diego, USA

\author{
Michele Zorzi \\ University of Padova
Department of Information Engineering
Via G. Gradenigo, 6/B, Padova, 35131, Italy
}

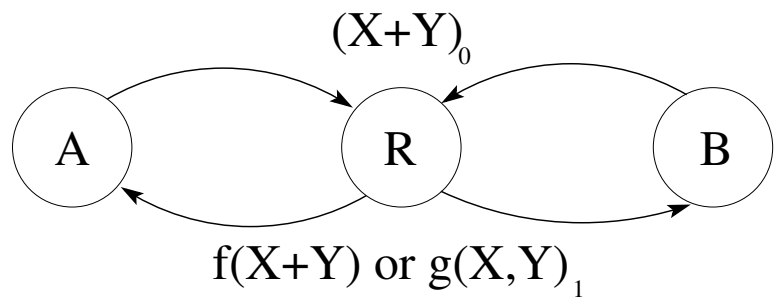

Fig. 1. The two way relay channel and physical layer NC. The subscripts $0 / 1$ stand for the time slot in which each packet is sent.

other hand, in decode-and-forward physical layer network coding (DF-PNC [6-9]), R decodes a linear combination L of X and Y directly from the analog superposition. This packet $\mathrm{L}$ is broadcast and $\mathrm{A}$ and $\mathrm{B}$ can remove their own frame from $\mathrm{L}$ to recover the desired data unit.

These two methods share the idea that $\mathrm{R}$ need not separately decode $\mathrm{X}$ and $\mathrm{Y}$, but just has to send a known function of them. However, a few important differences arise. First of all, in AF-PNC the signals do not need to be either frame synchronous or slot synchronous. Instead, to the best of the authors' knowledge, all published work on DF-PNC assumes that $\mathrm{X}$ and $\mathrm{Y}$ are symbol synchronous at $\mathrm{R}$, and in some cases [6,7] even phase synchronous (i.e., the complex-valued channels have the same phase). Such a symbol synchronization induces a certain structure in the received signals that eases the decoding process, for instance enabling the usage of sophisticated lattice-based schemes [6,7]. Lattice based schemes exhibit very good performance, but have been proven to work well only under the previous stringent hypothesis. [9] undertakes a somewhat more pragmatic approach, since phase synchronization is no longer needed (but symbol synchronization and flat fading are). Therefore, the channel gains can be different complex numbers.

Secondly, in AF-PNC, the noise at R is also amplified and hence $\mathrm{A}$ and $\mathrm{B}$ must face both their own noise and the forwarded (and amplified) noise in the received signal from $\mathrm{R}$. This can lead to a penalty of up to $5 \mathrm{~dB}$ between DF-PNC and AF-PNC [7].

This paper's aim is to propose a practical scheme for DF-PNC which is not constrained by some of the most stringent requirements of present DF-PNC techniques. In particular, our system enjoys the following properties:

1. The colliding signals are neither symbol nor phase synchronized;

2. The propagation environment can be frequency selective fading; 
3. The demodulation process yields soft output, so as to improve the performance of modern channel decoders. Instead, past work either jointly performs demodulation and channel decoding (but requires very specific channel codes) [6-8] or outputs only hard-decoded bits [9].

The rest of the paper is organized as follows. Sections 2 and 3 describe the scheme and its properties, respectively; the performance evaluation is carried out in Section 4 against DF-PNC with slot synchronization and AF-PNC. Finally, Section 5 draws the conclusions.

\section{SYSTEM MODEL AND SCHEME ARCHITECTURE}

The system architecture is depicted in Fig. 2. The first step is channel coding, which is assumed to be an $\operatorname{LDPC}$ on $\operatorname{GF}\left(2^{b}\right), b>0, b \in \mathbb{N}$, but the system would also work with other codes (such as turbo or convolutional codes). A non-binary finite field can be important because 1) non binary codes are more general than (and sometimes also superior to) their binary counterparts $[10,11]$ and 2) this enables the usage of NC coefficients in a larger field than GF(2). The latter element is essential in more complicated and realistic networks than the TWRC, since linear independence between different coded packets must be ensured. In the specific case of the TWRC, GF(2) is sufficient as far as network coding is concerned, since the relay just needs to send the XOR of X and Y. For this reason, the simulations in Section IV (which focus on the TWRC) adopt GF(2). We nonetheless remark that non-binary fields become essential in more general systems, leaving a more detailed investigation as a future work.

Let $\mathcal{X}$ and $\mathcal{Y}$ be the channel coded versions of $\mathrm{X}$ and $\mathrm{Y}$, respectively. Each codeword is divided into blocks each with as many bits as an Orthogonal Frequency Division Multiplexing (OFDM) symbol needs. Every block is split into Galois symbols and each Galois symbol is turned into $b$ bits and the coding phase is completed by mapping these bits into $K M$-ary QAM symbols $\mathcal{X}_{k}, \mathcal{Y}_{k}$, $b=K \log _{2}(M), 0 \leq k \leq K-1$, and performing OFDM modulation. All transmitters send their packets at the same time and hence the packets are linearly combined by the superposition on the wireless channel (as PNC requires).

Note that OFDM is a fundamental building block of our system and is essential for asynchronous PNC also in flat fading channels. Indeed, let us assume that $\mathrm{Y}$ is delayed with respect to $\mathrm{X}$. Since Y's signal will not be symbol synchronous with X's symbols, Y's delay can be represented as a two-tap frequency selective channel. The easiest way nowadays to cope with such a behavior is the usage of OFDM. As long as the relative delay $D$ between $\mathrm{X}$ and $\mathrm{Y}$ is smaller than the cyclic prefix length $\mathrm{CP}$, the channel is turned into a set of parallel flat fading channels. From another point of view, the two packets are slot synchronous but undergo a time varying channel $H_{i, n}, i \in\{A, B\}$, where $H_{i, n}$ is the frequency response of the channel impulse response $h_{i}$ as seen by the $n$-th subcarrier. Incidentally, the delay between the users generates a frequency selective channel and hence channel coding is even more essential because it enables to code over different fades and achieve frequency diversity.

The task of the receiver $\mathrm{R}$ is to compute a linear combination $Z=\alpha X+\beta Y$, where $\alpha$ and $\beta$ are arbitrary random coefficients in $\mathrm{GF}\left(2^{b}\right)$, based on the overlapping signals received from A and B with no synchronization requirements. This highlights two properties of our decoder: first, the algorithm can yield any linear combination of $\mathrm{X}$ and $\mathrm{Y}$, without decoding these two frames separately. Second, all previous work exploited the fact that the waveforms of $\mathcal{X}$ and $\mathcal{Y}$ that correspond to the same symbols in the codewords exactly match in time. Our decoder computes the desired linear combination without

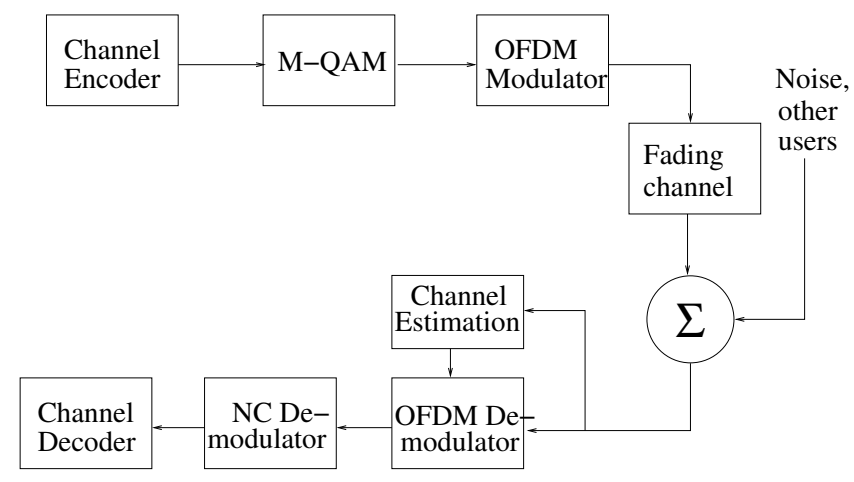

Fig. 2. The system architecture.

this requirement. Since $\alpha$ and $\beta$ are scalars in the same field of the channel code, we also have that:

$$
\mathcal{Z}=G Z=G(\alpha X+\beta Y)=\alpha \mathcal{X}+\beta \mathcal{Y}
$$

where $G$ is the channel encoding matrix. The channel is estimated and OFDM demodulation is performed. After this step, the received signal $\rho=\left[\rho_{0}, \rho_{1}, \ldots, \rho_{K-1}\right]$ can be represented as follows:

$$
\rho_{k}=H_{A, n(k)} \mathcal{X}_{k}+H_{B, n(k)} \mathcal{Y}_{k}+w_{n(k)}
$$

where $w_{n(k)}$ is complex, zero mean, circularly symmetric AWGN noise with variance $\sigma^{2}$ per dimension. The sampling time is left arbitrary, i.e., the receiver does not optimally sample either X or Y, since their respective sampling times do not coincide, in general. More on the sampling time will be said in the next section.

The heart of the decoding process is the NC demodulation. By $\mathrm{NC}$ demodulation we mean the process of directly estimating a linear combination $\mathcal{Z}$ of the transmitted information units and performing soft decoding rather than hard decoding. Hence, the output is the sequence of $\log$ likelihood ratios of a desired linear combination of $\mathcal{X}$ and $\mathcal{Y}$. Note that the NC demodulation is carried out on a Galois symbol by Galois symbol basis. As already pointed out in [7], it is essential to directly decode $\mathcal{Z}$, as opposed to separately estimating the individual frames ( $\mathcal{X}$ and $\mathcal{Y}$ in the previous example). Note that $\mathrm{A}$ and $\mathrm{B}$ just need $\mathrm{Z}$ to recover the intended frames and hence $\mathrm{R}$ has to decode only $\mathcal{Z}$ and not $\mathcal{X}$ and $\mathcal{Y}$ separately, thereby reducing the error probability.

For ease of notation, let us assume for the rest of the paper that $\mathcal{Z}, \mathcal{X}$ and $\mathcal{Y}$ indicate a generic Galois symbol of the LDPC codeword, rather than the whole codeword. The key problem (which addresses the third goal exposed in Section 1) is how to produce the soft bits for $\mathcal{Z}$. The output of the NC demodulator are the $\log$ Likelihood Ratios (LLRs) for the Galois symbols that compose the coded packet. The computation of the LLRs is equivalent to the evaluation of the probability that $\mathcal{Z}$ assumes one of its possible values $s_{j} \in\left\{0,1, \ldots, 2^{b}-1\right\}$. These probabilities can be assessed as follows. First, let us assume that the a posteriori probability $\mathrm{P}[(\mathcal{X}, \mathcal{Y}) \mid \rho]$ of each $(\mathcal{X}, \mathcal{Y})$ pair has been computed. Then, $\mathrm{P}[\mathcal{Z} \mid \rho]$ can be evaluated by marginalizing $\mathrm{P}[(\mathcal{X}, \mathcal{Y}) \mid \rho]$ over all ordered pairs $(\mathcal{X}, \mathcal{Y})$ that yield the same $\mathcal{Z}$ : 


$$
\begin{aligned}
p_{j}=P\left[\mathcal{Z}=s_{j} \mid \rho\right] & =\sum_{(\mathcal{X}, \mathcal{Y}): s_{j}=\alpha \mathcal{X}+\beta \mathcal{Y}} P[(\mathcal{X}, \mathcal{Y}) \mid \rho] \\
P[(\mathcal{X}, \mathcal{Y}) \mid \rho] & =P[\rho \mid(\mathcal{X}, \mathcal{Y})] \frac{P[(\mathcal{X}, \mathcal{Y})]}{P(\rho)} \\
P[\rho \mid(\mathcal{X}, \mathcal{Y})] & \propto e^{-\frac{1}{2 \sigma^{2}} \sum_{k=0}^{K-1}\left|\rho_{k}-H_{A, n(k)} \mathcal{X}_{k}-H_{B, n(k)} \mathcal{Y}_{k}\right|^{2}}
\end{aligned}
$$

The term $P[(\mathcal{X}, \mathcal{Y})]$ (the prior) is considered to be uniform (i.e., $\left.P[(\mathcal{X}, \mathcal{Y})]=2^{-2 b}\right)$ and $P(\rho)$ is a constant, thus they will be neglected hereafter. Eq. (3) can be computed exactly only by exhaustive enumeration of all the $2^{2 b}$ values of $P[(\mathcal{X}, \mathcal{Y}) \mid \rho]$. However, especially for large $b$, this is a hefty number. In addition, most of these probabilities are rather small, since $P[(\mathcal{X}, \mathcal{Y}) \mid \rho] \propto e^{-d(\rho, X, Y)^{2}}$, where $d(\rho, X, Y)$ is the Euclidean distance between the vector of received symbols $\rho$ and the expected samples if $(\mathcal{X}, \mathcal{Y})$ were the transmitted symbols. The task of picking the $C$ most likely pairs $(\mathcal{X}, \mathcal{Y})$ is equivalent to finding the closest couples $(\mathcal{X}, \mathcal{Y})$ to the received vector $\rho$, and such a task can be efficiently performed by means of soft-output sphere decoding $[12,13]$. In particular, a slightly modified version of [13] suited for this context is needed. First note that the constellation of valid points is $H_{A, n(k)} \mathcal{X}_{k}+H_{B, n(k)} \mathcal{Y}_{k}$, for all possible combinations of $\mathcal{X}_{k}, \mathcal{Y}_{k}$. We shall call this set the $k$-th expanded constellation. The modified sphere decoder works as follows:

1. The algorithm defines a sphere radius $r$. Its output is the set of pairs $(\mathcal{X}, \mathcal{Y})$ such that:

$$
\sum_{k=0}^{K-1}\left|\rho_{k}-H_{A, n(k)} \mathcal{X}_{k}-H_{B, n(k)} \mathcal{Y}_{k}\right|^{2}<r^{2}
$$

2. At any stage, the decoder keeps a list of the $C$ pairs with smallest metric (5). If fewer than $C$ pairs have been found so far (for instance at the beginning), the remaining elements in the list are fake placeholders with infinite metrics;

3. For the first signal sample $\rho_{0}$, the decoder finds all points in the expanded constellation for $k=0$ such that

$$
\left|\rho_{0}-H_{A, n(0)} \mathcal{X}_{0}-H_{B, n(0)} \mathcal{Y}_{0}\right|^{2}<r^{2}
$$

4. For each such point, the decoder finds all elements of the expanded constellation for $k=1$ such that

$$
\sum_{k=0}^{1}\left|\rho_{k}-H_{A, n(k)} \mathcal{X}_{k}-H_{B, n(k)} \mathcal{Y}_{k}\right|^{2}<r^{2}
$$

5. Repeat step 4 increasing the top index of the summation by one until the bottom is reached $(k=K-1)$. At any moment inside the loop, if $C$ pairs that satisfy (5) have been collected, assign to $r^{2}$ the metric of the furthest codeword among the $C$ gathered so far. This observation [13] makes condition (5) more and more stringent, thus pruning the set of possible candidates to evaluate.

After sphere decoding and marginalization (Eq. (3)), the channel decoder is given Z's soft decoded bits. The reception is completed by conventional channel decoding.

\section{REMARKS ON THE SYSTEM ARCHITECTURE}

In order to fully appreciate the importance of some architectural choices, a few remarks on the system design will be given below.

OFDM - The previous Section argued that OFDM is necessary to cope with the asynchronous nature of PNC. We now quantify the improvement of the system robustness to the relative delay between $\mathrm{X}$ and $\mathrm{Y}, D$, due to OFDM. First of all, we name the slot duration $T$. In slot synchronous DF-PNC (like $[7,8]$ ) it is reasonable to assume that the system works as long as $D$ is one fifth of $T$ because it is based on single carrier modulation. Such an estimate is based on the maximum tolerable delay spread in a single carrier system without equalization. Instead, in the proposed architecture, $D$ must be smaller than CP. If $N$ is the number of subcarriers, CP is usually $N T / 4$ or $N T / 5$. It is apparent that the maximum acceptable delay is about $N$ times larger for our system, and present wireless standards can employ, for instance, 64 subcarriers (like IEEE 802.11 [14]) or up to 2048 (as IEEE 802.16-2005 [15]). This suggests that our approach is about 2 or 3 orders of magnitude more resilient than conventional DF-PNC, just by using standard techniques in the right context. In addition we remark that the extension of DF-PNC to OFDM is not so obvious in this scenario, while being standard practice for the physical layer community. Indeed, OFDM is incompatible with some of the past DF-PNC architectures, such as latticebased schemes. We also note that OFDM naturally handles channels that are frequency selective. Let us call $\tau_{i}$ the delay spread of the $i$-th channel. The system can cope with an asynchronous system as long as $\max \left(\tau_{0}, D+\tau_{1}\right)$ is smaller than $\mathrm{CP}$. Hence, a frequency selective channel reduces the system robustness to the delay between the users' packets, but can be accommodated into our method in a very natural way, contrary to the other DF-PNC approaches.

Channel and Network Coding - Another important matter is that the NC demodulation process is performed before channel decoding. This should be the case, since the goal of this type of DF-PNC is to decode one rather than two packets. Hence, the channel decoder should be given as an input $\mathcal{Z}$ and not $\mathcal{X}$ and $\mathcal{Y}$ separately. In addition, the decoding of a single frame rather than two halves the computational burden on the channel decoder, while the cost is only (3) which implies just some fast additions. Incidentally, the marginalization in (3) reduces the error rate because multiple pairs $(\mathcal{X}, \mathcal{Y})$ contribute to the same $\mathcal{Z}$. Moreover, it is important that in Eq. (1) the $G$ matrix and the $\alpha, \beta$ coefficients can be swapped, so that to a certain linear combination of $\mathcal{X}$ and $\mathcal{Y}$ corresponds a linear combination with the same coefficients for $\mathrm{X}$ and $\mathrm{Y}$. The Galois elements $G$ and $\alpha, \beta$ can commute only if $\alpha, \beta$ belong to the same field of $G$ [16]. Hence, the field of the channel code is the limiting factor on the range of allowed linear combinations. For instance, if a binary code is used, the only possible value for $\alpha, \beta$ is 1 , while 0 is undesirable (it would imply not to consider one or both of the packets).

Sampling Time - The choice of the sampling time is left arbitrary. Such a decision is rather important: given a certain wireless channel in the analog domain, its energy in the digital domain (i.e., the sum of the squares of the absolute values of its samples) depends on the choice of the sampling time. For instance, if the shaping pulse is a rectangular waveform with unit amplitude and an ideal identity propagation channel is assumed, the waveforms $f_{x}(t), f_{y}(t)$ at the output of the matched filtered (for $\mathrm{X}$ and $\mathrm{Y}$ respectively) have a triangular shape (Fig. 3). If each signal is optimally sampled (at its peak), the energy of each digital channel is $T_{s}^{2}$, while if they are sampled with 


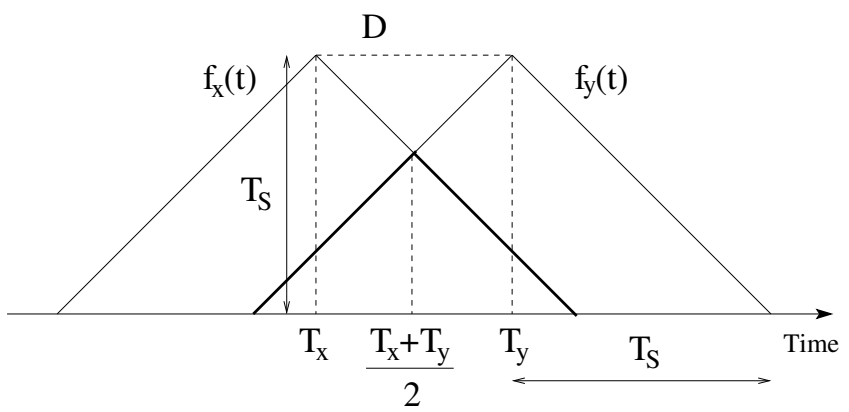

Fig. 3. Optimal choice of the sampling interval for equal channels. The minimum of the equivalent analog channels is the thick triangle.

an offset of $T_{s} / 2$ the energy is just $T_{s}^{2} / 2$. Since the two packets cannot be simultaneously optimally sampled, this loss of energy implies a Signal-to-Noise-Ratio (SNR) reduction which does not exist in conventional (synchronous) DF-PNC techniques. The optimization of such a choice is left as a topic for future work, but we propose the following simple heuristic: if the optimal sampling times for $\mathrm{X}$ and $\mathrm{Y}$ are $T_{x}+j T_{s}, T_{y}+j T_{s}, j \in \mathbb{Z}$, respectively, we propose to use $\left(T_{x}+T_{y}\right) / 2+j T_{s}$. This is a very simple choice which yields satisfactory results, as Section 4 will show. Furthermore, this strategy does not unfairly penalize either $\mathrm{X}$ or $\mathrm{Y}$ and actually attempts to maximize the minimum energy of $h_{A}$ and $h_{B}$ on average (i.e., assuming equal received powers). This is useful because it can be shown by a straightforward pairwise error probability analysis that the detection errors due to the weaker channel are those that dominate the error probability. We note that in the previous example of equal received powers and rectangular shaping pulses, the sampling time $\left(T_{x}+T_{y}\right) / 2$ does maximize the minimum energy (Fig. 3).

Network Coding coefficients - Finally, note that the choice of the NC coefficients is arbitrary. Such a property is exploited in the neighboring paper by $\mathrm{Pu}$ et al. [9], where the NC coefficients are used to optimize the decision regions at the demodulator so as to improve the bit error rate. This strategy is very attractive but works mainly in flat fading scenarios. The reason is that the same NC coefficients must be applied to all the symbols of the packets, and hence in a frequency selective channel a certain choice of $\alpha, \beta$ may be good for some subcarriers but not necessarily for the others. Hence, we leave as future work the adaptation of the NC coefficients to the channel. Nonetheless, we highlight again that our algorithm is able to decode an arbitrary linear combination of $\mathrm{X}$ and $\mathrm{Y}$, in spite of the fact the signals are superimposed in time and frequency.

\section{SIMULATION RESULTS}

The performance of the proposed architecture has been measured in terms of Bit Error Rate (BER) after channel decoding, which is computed by comparing the decoded $\mathrm{Z}$ against the expected linear combination $\alpha \mathrm{X}+\beta \mathrm{Y}$. In all cases, a 2/3 LDPC with 512 information bits is used. Given that the used OFDM system has 256 subcarriers, the 768 coded bits are split into 3 OFDM symbols. The Galois field is $\mathrm{GF}(2)$, and such a choice is motivated by the desire to investigate the performance of DF-PNC for well-established channel techniques and the sake of simplicity. Furthermore, in the TWRC, it is enough to pick the NC coefficients from $\mathrm{GF}(2)$, since $\mathrm{X} \oplus \mathrm{Y}$ is sufficient to recover the data units. The channels are subject to independent flat Rayleigh fading constant over all the received LDPC codeword, per-

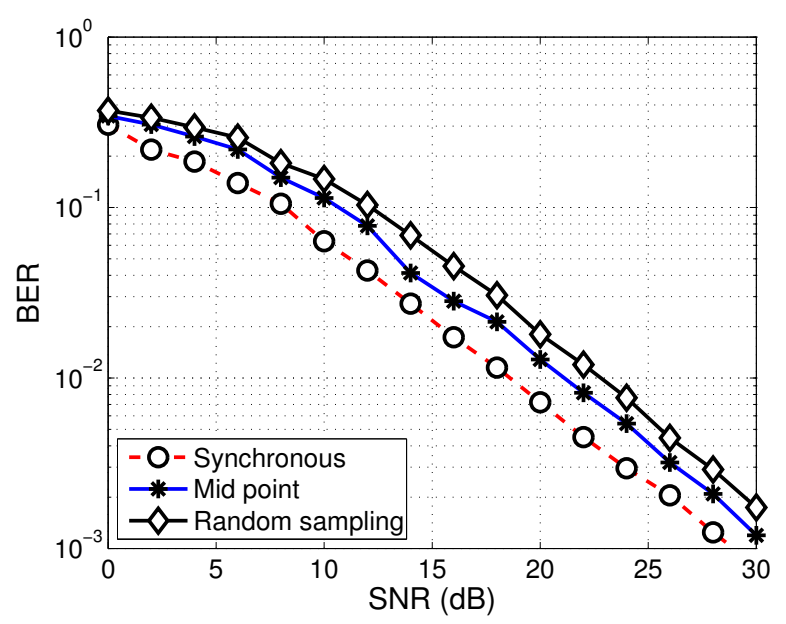

Fig. 4. Performance Comparison for the uplink of a TWRC with three different choices of the sampling time.

fect channel estimation is assumed throughout and enough simulations are run so as to collect several thousand bit errors for each SNR level. We do not incorporate frequency selective fading because this impairment just increases the relative delay of the colliding packets. In fact, frequency selective fading might even benefit our architecture, since it brings frequency diversity which can be effectively exploited by OFDM. Hence our results can be regarded as a pessimistic bound (from this point of view) on the achievable performance.

In the base scenario, $\mathrm{A}$ and $\mathrm{B}$ transmit their packets to $\mathrm{R}$ and $\mathrm{R}$ must correctly decode $\mathrm{Z}=\mathrm{X} \oplus \mathrm{Y}$. The studied design parameter is the sampling time and the performance of three different strategies is depicted in Fig. 4. In the first case, the signals are assumed to arrive synchronously and this curve provides a lower bound on the BER. In the second and third case, $\mathrm{X}$ and $\mathrm{Y}$ arrive asynchronously with a random delay uniformely distributed between 0 and the duration of the cyclic prefix CP. Hence, the probability density function of the mutual delay $D$ has a triangular shape between -CP and CP. In the former strategy, the sampling time is chosen as suggested in Section 2, i.e., it is the average of two neighboring sampling intervals for $\mathrm{X}$ and $\mathrm{Y}$. In the latter approach, the sampling time is completely random with respect to the colliding packets. As Fig. 4 shows, the "midpoint" strategy is about $2.5 \mathrm{~dB}$ away from the ideal synchronous scenario, while a choice of the sampling time completely oblivious of the frames' sampling instants would lose about $4.5 \mathrm{~dB}$. Hence, in spite of the simplicity of the proposed heuristics, almost half the gap from the optimal curve has been covered. It is reasonable that with more sophisticated ideas this gap can be further closed.

While no other DF-PNC apart from ours can be used for the twoway asynchronous relay channel, AF-PNC can be employed also for the asynchronous case and has actually been implemented in [4]. The basic idea is to enable each receiver A and B to subtract its own packet from the signal received from $\mathrm{R}$. Such a system is close to a successive interference cancellation scheme and can work also in the asynchronous case. ${ }^{1}$ In the TWRC, the performance metric is the average BER at A and B, i.e., A and B try to recover the other node's data unit and the BER is computed on the estimated frame. Fig. 5 shows that our architecture is about $2 \mathrm{~dB}$ away from the benchmark

\footnotetext{
${ }^{1}$ For AF-PNC, the issue of different sampling times is partly resolved by means of oversampling [4].
} 


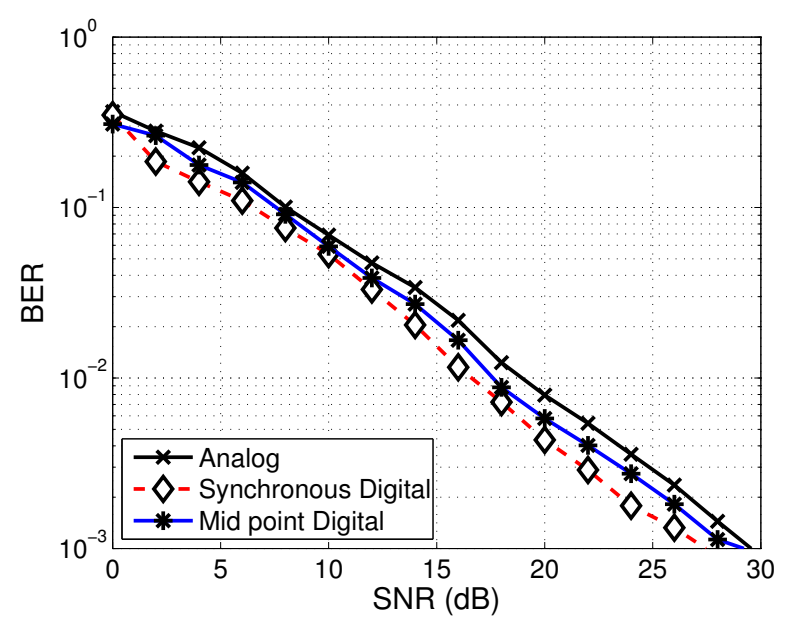

Fig. 5. Performance Comparison for the TWRC between synchronous DF-PNC, asynchronous DF-PNC and AF-PNC.

synchronous case and about $1 \mathrm{~dB}$ ahead of AF-PNC. Please note that Fig. 4 is not directly comparable to Fig. 5 because in the former the BER is computed at the relay, while in the latter it is an average of the BERs at A and B. Again, in spite of the early stage development of our system and the non negligible room for improvement, we have outperformed the benchmark practical PNC architecture. We also note that the gap between the ideal curve for DF-PNC and AF-PNC is around $3 \mathrm{~dB}$, which is less than the $5 \mathrm{~dB}$ predicted in [6]. The reason is that in [6] the channels are assumed to be identical in phase and magnitude. As suggested in Section 3, the performance in our architecture is limited by the weakest packet. Such a problem does not exists when the A-R and B-R channels are equal.

\section{CONCLUSIONS}

We have analyzed some of the implementation challenges of previous DF-PNC approaches and have proposed a simple yet effective strategy based on conventional physical layer techniques not yet fully appreciated in the context of wireless networking. The proposed architecture has been compared to AF-PNC, which is the other practical PNC approach.

The simulation results have shown that even a suboptimal implementation of our DF-PNC system concept is able to outperform AF-PNC. In addition to the performance improvements, other advantages are brought by this technique. For example, we expect DF-PNC to be more robust to channel estimation errors, since in AF-PNC the interference cancellation process is subject to these errors; instead DF-PNC is not affected, because A and B subtract their own digital packet ( $\mathrm{X}$ and $\mathrm{Y}$, respectively), rather than the estimate of the amplified analog signal at R. Moreover, AF-PNC needs oversampling to yield satisfactory performance, whereas our architecture still achieves good results without it. Furthermore, AF-PNC can transmit only a signal proportional to the sum of the colliding packets. Instead, DF-PNC can potentially yield any linear combination, and this flexibility is beneficial for $\mathrm{NC}$ in realistic topologies. Finally, AF-PNC does not work in frequency selective channels, while our proposal naturally accommodates these environments because of OFDM. All these points encourage additional investigation on this technique.
Future work involves the analysis of non-binary Galois fields, the study of the impact of channel estimation errors and the optimization of the sampling timing.

\section{ACKNOWLEDGMENT}

The first author would like to thank Hermann Bischl and Gianluigi Liva for the insightful discussions on the topic. This work is supported in part by the U.S. Army Research Office under the MultiUniversity Research Initiative (MURI) grant no. W911NF-04-10224.

\section{REFERENCES}

[1] S. Zhang, S. C. Liew, and P. P. Lam, "Physical-Layer Network Coding," in ACM MOBICOM, Los Angeles (CA, USA), Sep. 2006.

[2] P. Popovski and H. Yomo, "Bi-directional Amplification of Throughput in a Wireless Multi-Hop Network," in IEEE VTC Spring, Melbourne (Australia), May 2006.

[3] P. Popovski and H. Yomo, "Physical Network Coding in TwoWay Wireless Relay Channels," in IEEE ICC, Glasgow (UK), June 2007.

[4] S. Katti, S. Gollakota, and D. Katabi, "Embracing wireless interference: Analog network coding," in ACM SIGCOMM 2007, Kyoto (Japan), 27-31 Aug. 2007.

[5] S. Katti, I. Maric, A. Goldsmith, D. Katabi, and M. Medard, "Joint relaying and network coding in wireless networks," in IEEE ISIT 2007, Nice (France), 24-28 June 2007.

[6] B. Nazer and M. Gastpar, "Computation over multiple access channels," IEEE Transactions on Information Theory, vol. 53, no. 10, pp. 3498-3516, Oct. 2007.

[7] B. Nazer and M. Gastpar, "Compute-and-forward: Harnessing interference with structured codes," in IEEE ISIT, Toronto (Ontario, Canada), July 2008.

[8] I.-J. Baik and S.-Y. Chung, "Network coding for two-way relay channels using lattices," in IEEE ICC 08, Bejing (China), 1923 May 2008.

[9] W. Pu, C. Luo, B. Jiao, and F. Wu, "Natural network coding in multi-hop wireless networks," in IEEE ICC 2008, Bejing (China), June 2008.

[10] M. C. Davey and D. MacKay, "Low-Density Parity Check Codes over GF(q)," IEEE Trans. Inf. Theory, vol. 2, no. 6, pp. 165-167, June 1998.

[11] R. G. Gallager, Low-Density Parity-Check Codes, MIT Press, Cambridge (MA, USA), 1963.

[12] E. Viterbo and J. Boutros, "A Universal Lattice Code Decoder for Fading Channels," IEEE Trans. Inf. Theory, vol. 45, no. 5, pp. 1639-1642, July 1999.

[13] B. M. Hochwald and S. ten Brink, "Achieving near-capacity on a multiple-antenna channel," IEEE Trans. Commun., vol. 51, no. 3, pp. 389 - 399, Mar. 2003.

[14] IEEE Standards Department, ANSI / IEEE Standard 802.11, IEEE Press, 1999.

[15] IEEE Standards Department, ANSI / IEEE Standard 802.16e, IEEE Press, 2005.

[16] R. Blahut, Theory and practice of error control codes, Addison Wesley, Reading (MA, USA), 1983. 